\title{
A novel fast-neutron detector concept for energy-selective imaging and imaging spectroscopy
}

\author{
M. Cortesi, ${ }^{1,2}$ V. Dangendorf, ${ }^{3}$ R. Zboray, ${ }^{2}$ and H.-M. Prasser ${ }^{1,2}$ \\ ${ }^{1}$ Nuclear Energy and Safety Research Department, Paul Scherrer Institut, Villigen PSI 5234, Switzerland \\ ${ }^{2}$ Mechanical Engineering Department, Swiss Federal Institute of Technology, Zurich 8092, Switzerland \\ ${ }^{3}$ Ion and Neutron Radiation Department, Physikalisch-Technische Bundesanstalt, \\ Braunschweig 38116, Germany
}

(Received 17 April 2014; accepted 4 July 2014; published online 25 July 2014)

\begin{abstract}
We present and discuss the operational principle of a new fast-neutron detector concept suitable for either energy-selective imaging or for imaging spectroscopy. The detector is comprised of a series of energy-selective stacks of converter foils immersed in a noble-gas based mixture, coupled to a position-sensitive charge readout. Each foil in the various stacks is made of two layers of different thicknesses, fastened together: a hydrogen-rich (plastic) layer for neutron-to-proton conversion, and a hydrogen-free coating to selectively stop/absorb the recoil protons below a certain energy cut-off. The neutron-induced recoil protons, that escape the converter foils, release ionization electrons in the gas gaps between consecutive foils. The electrons are then drifted towards and localized by a positionsensitive charge amplification and readout stage. Comparison of the images detected by stacks with different energy cut-offs allows energy-selective imaging. Neutron energy spectrometry is realized by analyzing the responses of a sufficient large number of stacks of different energy response and unfolding techniques. In this paper, we present the results of computer simulation studies and discuss the expected performance of the new detector concept. Potential applications in various fields are also briefly discussed, in particularly, the application of energy-selective fast-neutron imaging for nuclear safeguards application, with the aim of determining the plutonium content in Mixed Oxide (MOX) fuels. () 2014 AIP Publishing LLC. [http://dx.doi.org/10.1063/1.4890392]
\end{abstract}

\section{INTRODUCTION}

Fast-neutron imaging combined with energy spectrometry can provide a wealth of information as a non-destructive measurement technique for screening and elemental characterization of bulk material, to support basic research, industrial, medical, and security related activities. ${ }^{1}$ However, combined spectroscopy and imaging with fast-neutrons poses a variety of challenges for many practical applications: some applications require large-area detectors (up to $\mathrm{m}^{2}$, e.g., in container screening or portal monitors); it should be made of simple and cost-effective hardware components; it needs to provide good imaging capability (spatial resolution of the order of $\mathrm{mm}$ ); and it should be insensitive to gamma radiation. This has to be achieved within the limitations posed by the comparably low cross-sections of the interaction of fastneutrons with matter and with the requirement of reasonably high detection efficiency (few tens of percent), which can be achieved only with converters of several $\mathrm{cm}$ effective thickness. The demands placed upon new fast-neutron detection technologies by the various scientific and industrial applications naturally increase with the complexity and the high neutron flux required in future systems.

One of the most interesting concepts of fast-neutron detectors for imaging spectroscopy, proposed in the last decades by several groups, are based on a fine-structured matrix of neutron converters (scintillator), such as plastic fibers (or capillary tubes) filled with liquid scintillator ${ }^{2-4}$ or an organic luminescent matrix screen; ${ }^{5}$ by recording the images of the ionization tracks of the recoil protons, the detector allows the energy and direction of the impinging neutron to be reconstructed. The photo-sensor can be either a CCD camera, with limited field of view, ${ }^{2}$ or a complicated and expensive position-sensitive gaseous photomultiplier. ${ }^{6}$ Energy determination by time of flight (TOF) measurements ${ }^{7}$ can be also achieved for fast neutrons by using a pulsed neutron generator: ${ }^{8}$ however, in this approach the high cost are often prohibitive due to low turnaround in most of the industrial applications, and the available neutron flux is exceedingly reduced due to the low duty cycles of the beam.

A cost-effective, large-area, two-dimensional imaging detector may be realized with a hydrogen-rich converter coupled to a position-sensitive charge readout based on Micro-Pattern Gaseous Detector (MPDG) technologies (e.g., Gaseous Electron Multiplier- GEM). ${ }^{9,10}$ Such detectors are relatively simple and they can potentially fulfill many of the demands; however, they possess no energy selectivity and spectroscopic capabilities, and the detection efficiency is rather poor.

In this work, we present an innovative concept of energyselective fast-neutron imaging, which is based on these converter foils coupled to MPDGs, but overcome the limitation in spectroscopy and efficiency of the existing technique. The conceptual idea of the detector is a further development of a previous concept proposed by our group, which aimed at developing a high-efficiency, large-area, fast-neutron imaging detector for fan-beam tomography. ${ }^{11,12}$

A potential application of the proposed new detector concept is fast-neutron imaging spectroscopy: the latter is 
achieved with a converter made of stacks of foils whose thicknesses, of both the converter layers and of the energy-selective coatings, is gradually increased; the specific responses of the converter to different neutron energies allows spectroscopy to be performed by unfolding analysis. In this work, the fastneutron spectrum unfolding problem is solved by the nonnegative least-squares approach.

A far simpler practical application is achieved when the energy spectrum of the impinging neutron beam is a linear combination of few well-defined spectral components; in this case, only a few parameters (e.g., the magnitude of each component) have to be determined to solve the system of equations for reconstructing the original spectrum. The unfolding problem is disentangled by the aid of selected response functions, given by a small number of energy-selective stacks which have to be chosen to be sensitive to the known constituents of the neutron spectrum to be determined.

In this work, we present in detail the operational principle of the new detection concept in view of possible applications as an energy-selective neutron imaging detector and for neutron imaging spectroscopy. In particular, we discuss the example of characterization and quality control of Mixed Oxide (MOX) fuel by spectroscopic analysis of the plutonium content using an energy-selective imaging detector model; the latter consists of a small number of energy-selective stacks. The discussion is based on results of response calculations using the MCNPX code $^{13}$ and unfolding the calculated responses which are compared to the known input spectra.

\section{DETECTOR CONCEPT}

The conceptual design of the detector comprises of several energy-selective stacks of converter foils, several millimeters height, coupled to a two-dimensional positionsensitive gas detector. The foils are aligned perpendicular to the impinging neutron flux (Figure 1), and separated by sub-millimeter thick gas-filled gaps (typically $0.7 \mathrm{~mm}$ ). The position-sensitive gas detector may be based on MultiWire Proportional Chamber (MWPC) or on Micro-Pattern Gaseous Detector (MPGD) technology, ${ }^{14}$ such as standard Gaseous Electron Multiplier (GEM) ${ }_{15}^{15}$ Thick Gaseous Electron Multiplier (THGEM), ${ }^{16}$ Micro-MEsh Gaseous detector (MICROMEGAS), ${ }^{17}$ or other similar detector structures.
Each converter stack encloses a number of foils, of equal geometrical dimensions, made of two layers fastened together: a hydrogen rich layer for neutron-to-proton conversion, and an energy-selective coating. The thickness of both the hydrogenous converter layers and of the energy-selective coatings is progressively increased with the increasing penetration of the neutrons into the detector, in such a way that low energy neutrons are preferably detected in the first layers while in the deeper layers the efficiency for detecting higher energy neutrons is successively increased. Collision processes, mainly elastic n-p scattering, may occur between impinging neutrons and hydrogen atoms inside a converter layer, in one of the many stacks along the neutron direction. The recoil proton enters in the energy-selective coating and is slowed down and, depending on its energy, it might be completely stopped. According to the thickness of the energy selective coating and the charged particle's recoil energy, only those above a certain threshold, and within a certain angular range can escape the composite foil converter and ionize the gas in the gap between two consecutive foils. Upon application of a suitable electric field (around $1 \mathrm{kV} / \mathrm{cm}$ ), the ionization electrons are then drifted in the gas gaps towards the two-dimensional position-sensitive gas filled detector: e.g., the MPGD utilizes gas avalanche electron multiplication, which enables detection and localization of the small, neutron-induced ionization by a pixilated, charge sharing or delay-line anode readout. By arranging several stacks of foils with increasing foils thicknesses and different energyselective coating layers, neutrons of a certain energy will predominantly being detected in certain locations inside the foil assembly, according to the thicknesses of the converter and the energy-selective coating; e.g., low energy neutrons are detected predominantly in the first few sectors of thin radiator layers and none or rather thin energy selective coatings, where low energy recoil protons may escape the converter foil. On the other hand, stacks comprised of thick radiators, with thick coatings, allow only higher energetic recoil protons from faster neutrons to escape and being detected. In this configuration, each specific foil in the various stacks has a different response function to the various impinging neutron energies. Therefore, the one-dimensional profile of the counting statistics parallel to the impinging neutron beam is characteristic to a certain neutron spectrum.

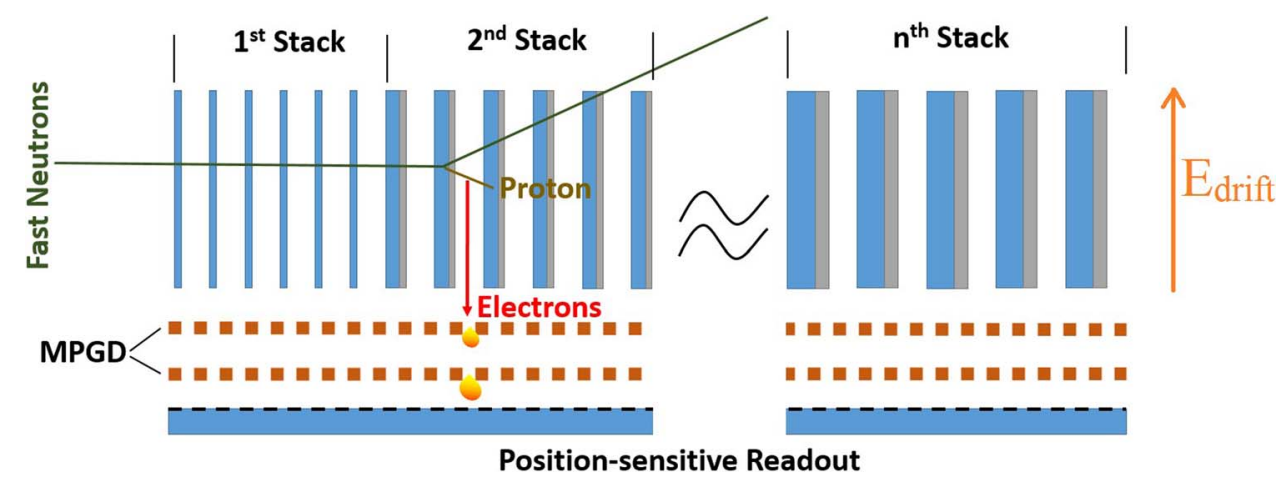

FIG. 1. Schematic drawing of the energy-selective, fast-neutron imaging detector. The latter is comprised of two parts: (1) an assembly of several energyselective stacks of converter foils (each of them made of a hydrogenous layer for neutron-to-proton conversion, optionally coated by a hydrogen-free layer to stop the proton beyond a certain cut-off energy and (2) a position-sensitive charge readout (e.g., based on MPGD technology). 
In order to achieve the highest neutron-to-proton conversion efficiency, the converter layer is made of a material with a high content of hydrogen (e.g., polyethylene or polypropylene). The energy-selective coating serves as a proton energy cutoff, and it is made of a material possessing a preferably high proton stopping power and no hydrogen. A good material family for coating are metal oxides, such as $\mathrm{SiO} 2$ or Hafnia $\left(\mathrm{HfO}_{2}\right)$. Hafnia has gained considerable attention in the microelectronic industry in order to replace $\mathrm{SiO}_{2}$ as capacitor dielectric material, at first because of its high permittivity, related high breakdown field, and to sufficient thermodynamic stability with respect $\mathrm{Si} .{ }^{18}$ In addition, due to its high atomic number and high mass density $\left(9.68 \mathrm{~g} / \mathrm{cm}^{3}\right)$, Hafnia is ideal as energy-selective coating. Alumina $\left(\mathrm{Al}_{2} \mathrm{O}_{3}\right)$ or Silica $\left(\mathrm{SiO}_{2}\right)$ may be also a possible solution for the coating layer. On the one hand, in order to keep a good electric field geometry inside the gas gap between two consecutive foils, necessary for a high electron transport efficiency from the converter gas gap to the position- sensitive charge readout, the coating should be non-conductive. On the other hand, to avoid charging up of the foils surface, which may cause degradation of the electron transfer efficiency, the coating should be slightly conducting: so the surface charge may be removed and the electric field lines in the gas gap should be straight and parallel to the surface of the converter foils. ${ }^{19}$ Good electron transfer efficiency (ETE), with minimal diffusioninduced losses, was demonstrated in narrow gas gap between (resistive) polymer foils, several $\mathrm{mm}$ high, and was reported in a recent work. ${ }^{19}$ A similar ETE is expected for the energyselective foils proposed here; however, possible charging up effect on the aforementioned metal oxide coating may arise in the case of operation at high counting rate, with the possibility of significant loss of ETE. To overcome this problem, the possibility of using resistive material for the energy-selective coating, including thermally evaporated $\mathrm{Ge}$ or Si layers is investigated.

\section{SIMULATION OF THE DETECTOR RESPONSE}

\section{A. Fast-neutron spectroscopy}

Neutron spectroscopy is realized by processing the image obtained from a detector whose converter is made of several energy-selective stacks, capable of producing different detector response functions for different neutron energy ranges. The relation between the count rates in the various converter stacks $\left(\mathrm{N}_{\mathrm{i}}\right)$ and the energy spectrum of the impinging neutron beam $\left(\mathrm{F}_{\mathrm{j}}\right)$ is uniquely characterized by the detector response matrix $R_{i j}$, expressed through the Fredholm integral of the first kind in its discretized numerical form:

$$
\mathrm{N}_{\mathrm{i}}=\sum_{\mathrm{j}=1}^{\mathrm{M}} \mathrm{R}_{\mathrm{ij}} \mathrm{F}_{\mathrm{j}},
$$

where $\mathrm{N}_{\mathrm{i}}$ is the flux count rate detected in the $i$ th stack, $\mathrm{F}_{\mathrm{j}}$ is the incident neutron flux of the jth energy bin (to be determined) and the $\mathrm{R}_{\mathrm{ij}}$ is the response (detection efficiency) of the $i$ th stack to neutrons in the $j$ th energy bin.

Experimental or numerical uncertainties might exist in the response matrix (R) and in the detected count rate $\mathrm{N}$, both affecting significantly the solution of the ill-posed problem of equation (1). A large numbers of techniques and methods for unfolding energy spectra have been developed to optimize the solution of Eq. (1), including generic algorithm, regularization, parameterization, and iterative procedures. In this work, we apply an optimization method by solving the least square problem of the form

$$
\min _{F_{j}}\left(N_{i}-\sum_{j=1}^{M} R_{i j} F_{j}\right)
$$

for each of the $i$ th stack. The task is to find the non-negative amplitude coefficients $\left(\mathrm{F}_{\mathrm{j}}\right)$ for each of the jth energy components of the impinging neutron spectrum.

In order to illustrate the operational mechanism of the new detector concept, we have modeled the performance of an energy-selective converter by MCNPX simulations, and unfolded a few spectra using the standard non-negative least square code of Matlab. The converter is designed to be sensitive to neutron energies in the range of $2-20 \mathrm{MeV}$ and it is made of 27 different stacks, each of them consists of a different number of converter foils (polyethylene/Hafnia). The thickness of both the converter layer and coating was increased, from $0.1 \mathrm{~mm}$ to $0.24 \mathrm{~mm}$ for the polyethylene layers and from 0 to $0.08 \mathrm{~mm}$ for the energy-selective Hafnia coating. The gas gap between the neighbor foils is $0.5 \mathrm{~mm}$ in each stack and it is filled with $\mathrm{Ne}$-based mixtures (i.e., $\mathrm{Ne} / 5 \% \mathrm{CF}_{4}$ or $\left.\mathrm{Ne} / 5 \% \mathrm{CH}_{4}\right)-\mathrm{Ne}$ mixtures are known to provide a relative high electron multiplication gain as well as high dynamic, ${ }^{20}$ allowing the ionization of the escaping proton to be detected by the position-sensitive gaseous detector. It follows that the full converter structure is composed of 215 foils, arranged on a $30 \mathrm{~cm}$ long. In our simulation, the detector active area was designed to be $10 \mathrm{~cm}$ wide. Mono-energetic neutron irradiations were simulated to compute the detector response matrix of the detector efficiency of each stack as function as the impinging neutron energy.

Figure 2 shows the responses (detection efficiencies) computed for each converter foil for neutron energies of 4 , $6,8,10$, and $12 \mathrm{MeV}$. The detection efficiency is defined as the number of recoil protons that escape the foils into the

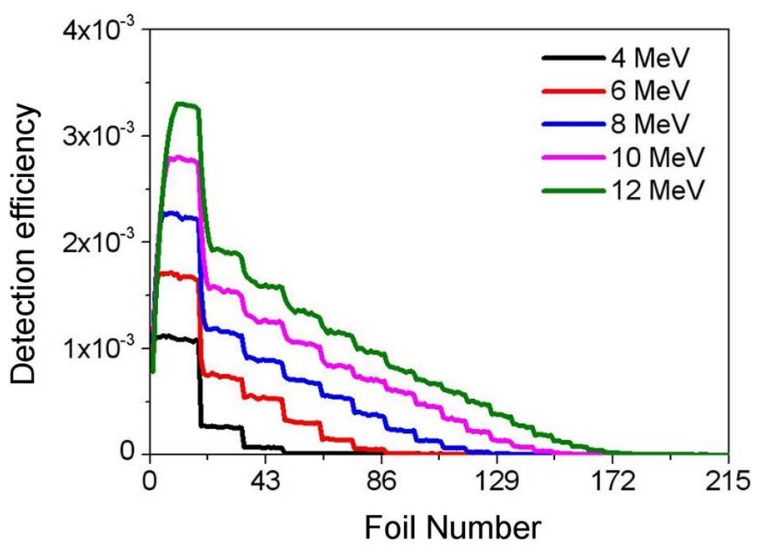

FIG. 2. Computer-simulated response functions (detector efficiency) of each energy-selective converter foil, as function of the energy of the impinging neutron beams $(4,6,8,10$, and $12 \mathrm{MeV})$. 
(a)

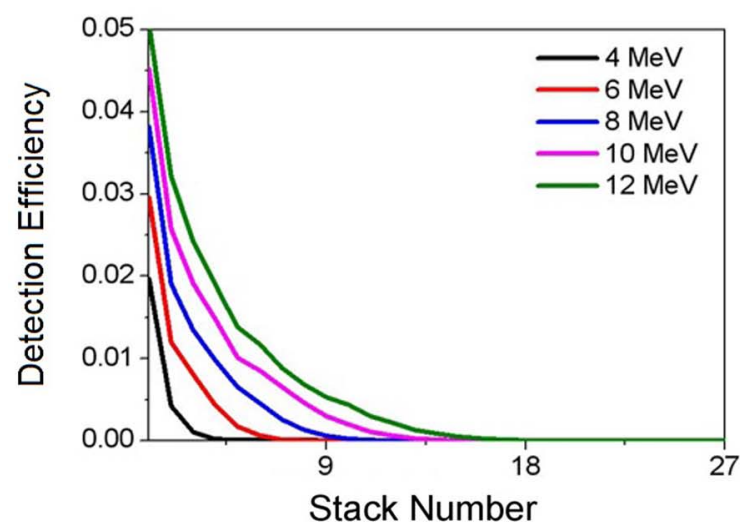

(b)

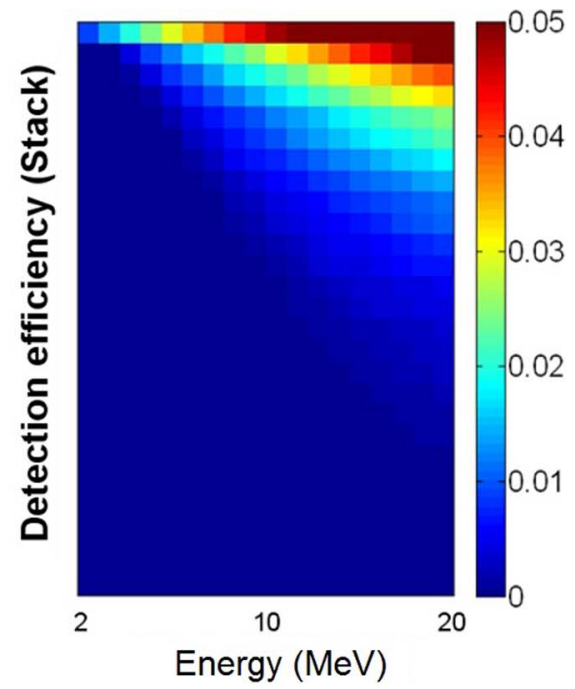

FIG. 3. (a) Computer-simulated response functions (detector efficiency) of each stack of energy-selective foils, as function of the energy of the impinging neutron beams $(4,6,8,10$, and $12 \mathrm{MeV})$. The detection efficiency of a stack is the sum of the detection efficiency of all the converter foils contained in that stack. (b) Computer-simulated detection response matrix used for spectral unfolding. The dimension of the matrix are 27 detection efficiency bins (corresponding to energy-selective stacks) and 19 neutron energy bins (1 MeV width).

gas gaps, thereby releasing a sufficient amount of ionization electrons which can be consequently detected by the positionsensitive charge readout, normalized by the total number of impinging neutrons. Notice that the "total" detection efficiency of the method will be given by the responses of Figure 2, multiplied by the efficiency of transport the ionization electrons along the thin gas gap ${ }^{19}$ and the efficiency of the position-sensitive detector. Minimum electron losses (ETE of the order of 95\%) were achieved along narrow gas gap for small drift length $(<10 \mathrm{~mm})$. In addition, the spectrum of energy deposited by the recoiled proton is similar to the energy distribution released by minimum ionizing particle (Landau distribution $)^{19}$ — detection efficiency close to $100 \%$ were achieved for MIP with MPGD technologies.

Instead of considering the response of each individual foil, a more simple analysis and a lower number of variable involved, may be achieved considering the responses generated by the each stacks; the response of a stack to a certain neutron energy is computed as the cumulative sum of the detection efficiencies of all the foils assembled in that stack. For instance, Fig. 3(a)) illustrates the detector responses provided by the stacks to few energy values and it was computed from data depicted in Fig. 2. As the energy resolution through spectrum unfolding depends on the size of the response matrix, a higher-resolution response matrix, computed by simulating systematically a larger and denser energy range, can increase the performance. Figure 3(b)) illustrates an example of response matrix constructed with a dimensional resolution of 27 detection efficiency bins (converter stacks) and 19 neutron energy bins (1 MeV bin width).

Examples of recovering of few simple spectra by a simulated measurement with the detector and unfolding its response are shown in Figure 4. The response of the detector to the input spectra (red line graphs) were obtained with MCNPX simulations using the detector model described above, generating a total of $10^{6}$ neutron's histories (total detection ef- ficiency of few percentage). The unfolding process was computed using the response matrix depicted in Figure 4(b)). The unfolded spectra (black-dash graphs) are compared to the input spectra used in the simulation red-line graphs); in this context, the idea of the present exercise was to assume simple spectra as inputs for the MCNP simulation, in order to test the method and evaluate the spectroscopic performance of the energy-selective converter. The first two spectra were used to test the resolving power of the in terms of discrete energy separated by small energy gaps, while the last one resemble a continuo decay spectrum, similar to typical fission spectra. In Figure 4, both the simulated spectra and the reference ones were normalized to their integral.

The discrepancy between the unfolded spectra and the reference ones, shown in Figure 4, are mainly caused by poor statistics of the MCNPX simulations and by the poor performance of the applied least-square method. Notice that bad statistics affects significantly the uncertainty in the response matrix $\mathrm{R}$ and the counting rate computed in each stack, affecting the result of the unfolding process to a large extent. A more precise energy spectra reconstruction may be obtained by applying more sophisticated codes and algorithm for the spectrum unfolding computation, including sequential least square method, artificial neutron network, Bayesian unfolding approach, etc.

\section{B. Characterization of MOX fuel}

As a matter of fact, the complexity of the approach to the spectral unfolding problem depends largely on the quality and on the robustness of the a priori information about the solution. In case the spectrum of the impinging neutron beam can be decomposed in a small number of well-known spectral components, a stable solution may be achieved by determining only a few parameters, i.e., the amplitude coefficients of each spectral component, whose shape is already known. An 

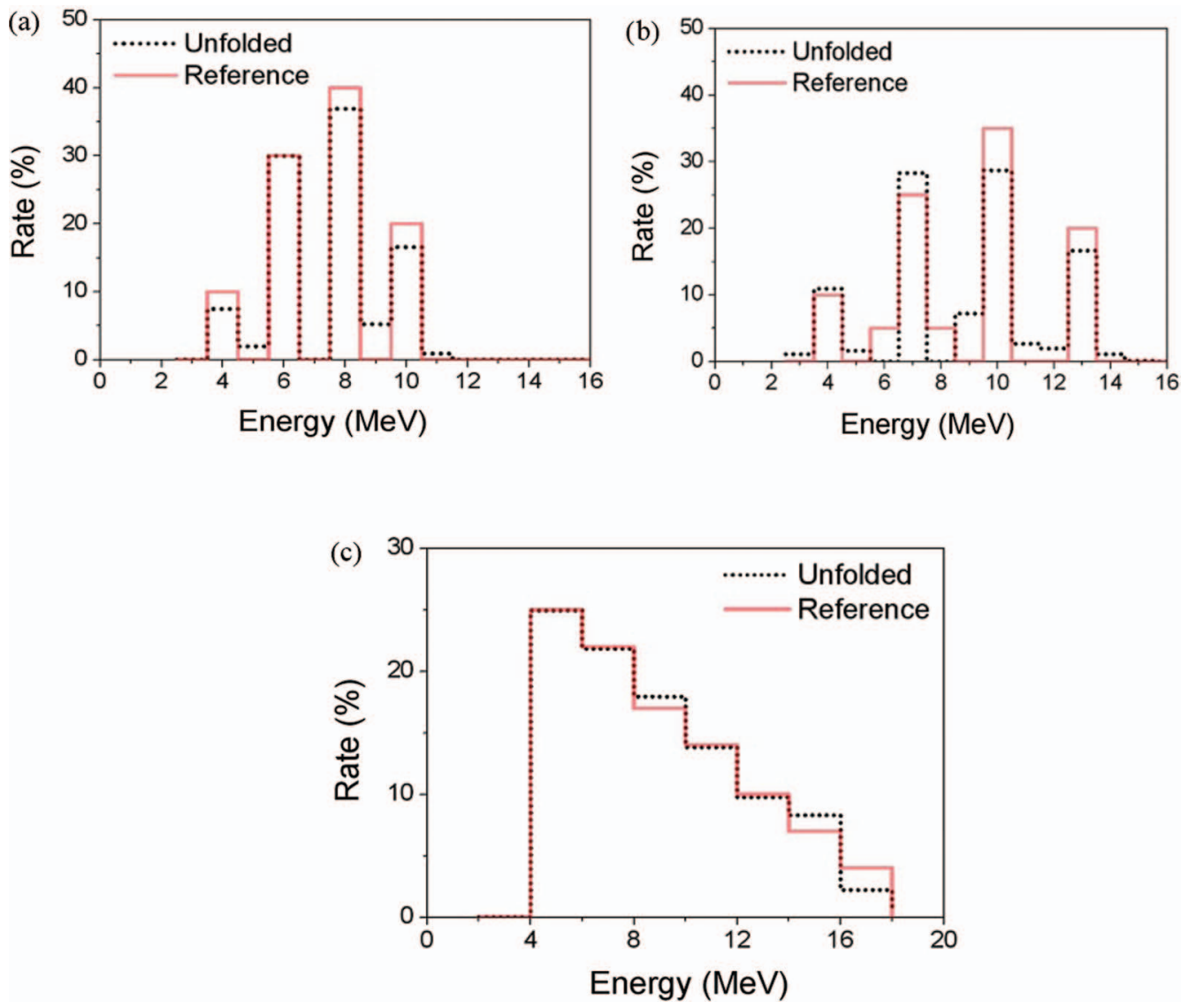

FIG. 4. Examples of unfolded energy spectra (black graphs) compared to the reference energy spectra (red graph), resolved by simulating the response of an energy-selective converter and by application of least-square technique.

example is the spectrum of MOX fuel which is composed of two constituents, a fission spectrum and a "(alpha,n)" component whose relative contribution has to be determined. The structure of the energy-selective converter can then be significantly simplified and being reduced to a few stacks, whose response functions are sensitive to the differences between the various spectral components.

Spectroscopy on MOX fuel is indeed a promising application of the present energy-selective detector .A typical problem in nuclear safeguarding is the quality control of fissile mass in nuclear material by determining the plutonium content in Mixed Oxide (MOX) fuel. MOX fuel usually consists of two phases, $\mathrm{UO}_{2}$ and $\mathrm{PuO}_{2}$, and/or a single phase solid solution $(\mathrm{U}, \mathrm{Pu}) \mathrm{O}_{2}$, where the uranium and plutonium are reprocessed from spent nuclear fuel and used to manufacture new fuel assemblies. The content of $\mathrm{PuO}_{2}$ may vary from $1.5 \mathrm{wt}$. \% to $25-30 \mathrm{wt}$. \% depending on the type of nuclear reactor; the principal isotopes of plutonium contained in MOX are the $238 \mathrm{Pu}, 239 \mathrm{Pu}, 240 \mathrm{Pu}, 241 \mathrm{Pu}$, and $242 \mathrm{Pu}$.

The characterization of the MOX fuel by passive neutron measurements, in an experimental setup similar to the one depicted in Fig. 5, is possible because of the presence of a significant amount of $240 \mathrm{Pu}$ (strong spontaneous fission source, with an emission of around 1000 neutrons per second per gram) and other alpha-emitting isotopes via $(\alpha, \mathrm{n})$ reactions with oxygen enclosed in the fuel matrix. ${ }^{21}$
While the spontaneous fission neutrons (SF) from 240Pu are emitted with a continuous energy spectrum, well represented by the Watt distribution ${ }^{22}$ with a most probable value (MPV) of $0.8 \mathrm{MeV}$, the alpha reaction on oxygen produces a neutron emission spectra (AN) peaked at around $2.3 \mathrm{MeV}$ and does not extend to energies higher than around $4 \mathrm{MeV}$ - the contribution of fission neutrons is far more dominant at ener-

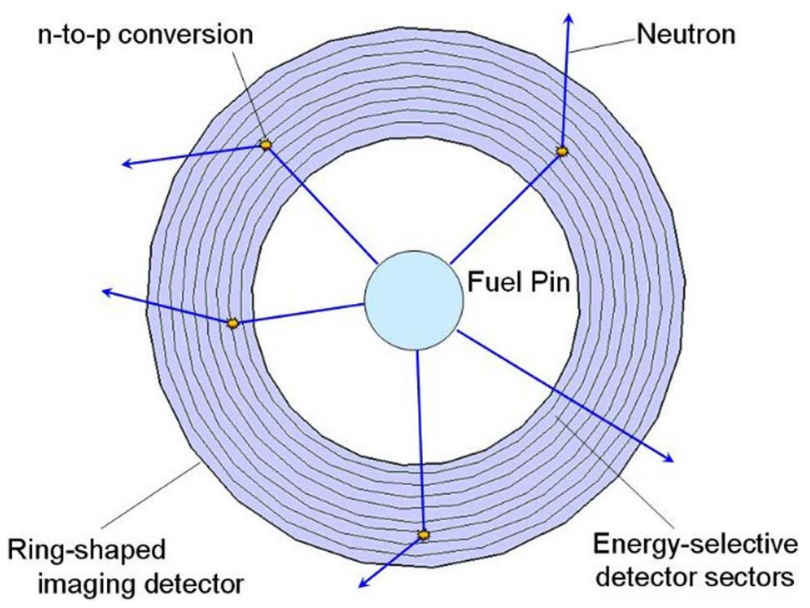

FIG. 5. Schematic drawing of the setup for passive analysis of neutron source (i.e., special nuclear material, nuclear fuel pin, etc.) using the energyselective imaging detector. 

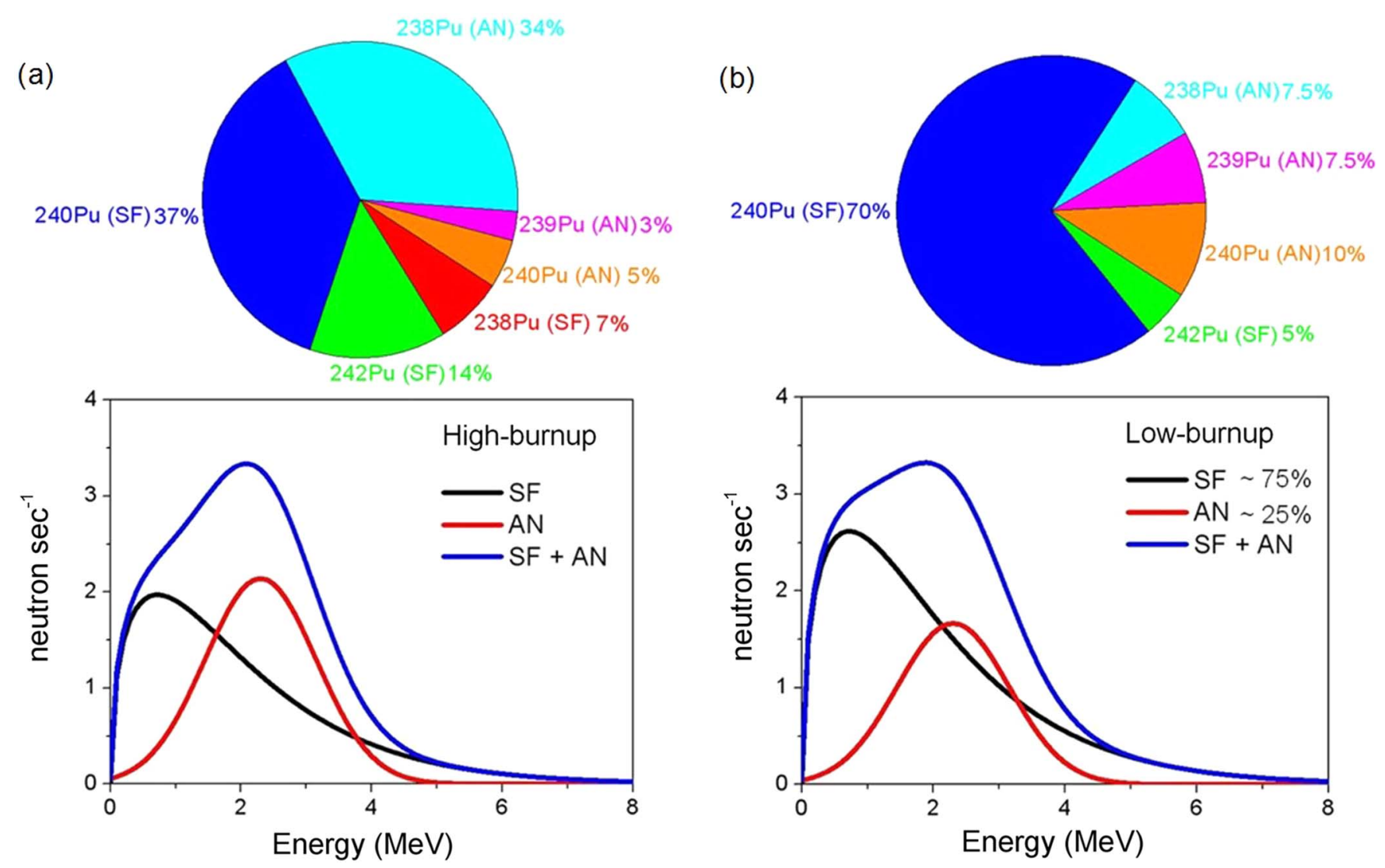

FIG. 6. Example of neutron emission spectra from high-burnup (left) and low-burnup (right) MOX fuel. ${ }^{22}$ The total emission spectra (blue graph) are the sum of spontaneous fission (SF) spectrum (Watt distribution), mainly from 240Pu decay (black graph), and $(\alpha, \mathrm{n})$ reaction spectrum (AN); the latter represented by a wide Gaussian distribution centered at around $2.3 \mathrm{MeV}$.

gies above $3.5 \mathrm{MeV}$. In the case of the high burn-up fuels, the $(\alpha, \mathrm{n})$ contribution to the total neutron emission is more significant than that of the low burn-up ones, due to the higher $238 \mathrm{Pu}$ and $240 \mathrm{Pu}$ content from longer irradiation times. ${ }^{23}$ This is an important feature because the energy-selective capability allows the two neutron emission spectra components to be discriminated, and thus to estimate the amount of fuel burn-up. Figure 6 shows typical total emission spectra from low-burnup and high-burnup MOX fuel, ${ }^{23}$ used as source input for performing our Monte Carlo simulations.

We have evaluated the capability of measuring the contribution of the two different neutron emission spectra in MOX fuels, by simulating a dedicated two-stack converter geometry and by processing the resulting detector responses. The converter, designed to be sensitive to the small energy difference between the fission and $(\alpha, \mathrm{n})$ energy spectra, in the range between 2 and $6 \mathrm{MeV}$, consists of two stacks (200 foils each): the first stack are made of Polyethylene foils of $100 \mu \mathrm{m}$ thickness and Hafnia coating of $40 \mu \mathrm{m}$; the second stack the Polyethylene layer and the Hafnia coatings are $300 \mu \mathrm{m}$ and $60 \mu \mathrm{m}$ thick, respectively.

Figure 7 illustrates the detector responses of the simulated converter, described in terms of detection efficiency of the converter foils, for both spontaneous fission spectrum (Watt distribution) and $(\alpha, \mathrm{n})$ neutron energy spectrum, respectively, black and red graph. The graphs also show the total detection efficiencies for each stack (denoted as $\varepsilon$ - subscript refer to the stack number while superscripts refer to the spec- tra, 1 for $\mathrm{SF}$ and 2 for $\mathrm{AN}$ ), computed as sum of the detection efficiency of all the foils in that stack. Notice that the ratio of detection efficiencies of the first stack $\left(\varepsilon_{1}^{1} / \varepsilon_{1}^{2}\right)$ is equal to 1.7 , while for the second stack $\left(\varepsilon_{2}^{1} / \varepsilon_{2}^{2}\right)$ is around 2: the difference between these two values is a measure of the sensitivity of the

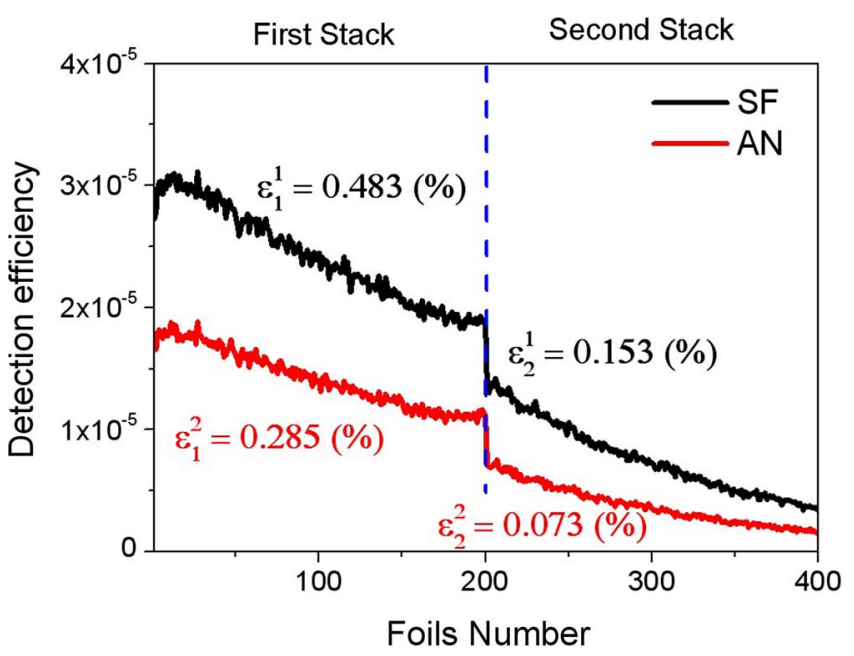

FIG. 7. Neutron detection efficiency computed for each converter foil, by simulating the irradiation of a two-stack converter (geometry described in the text) with neutrons of a spectral distribution composed of a spontaneous fission (SF) and an $(\alpha, \mathrm{n})$ (A.N) spectrum. Direction of the neutron beam is from foil No. 1 towards foils of higher numbers. 


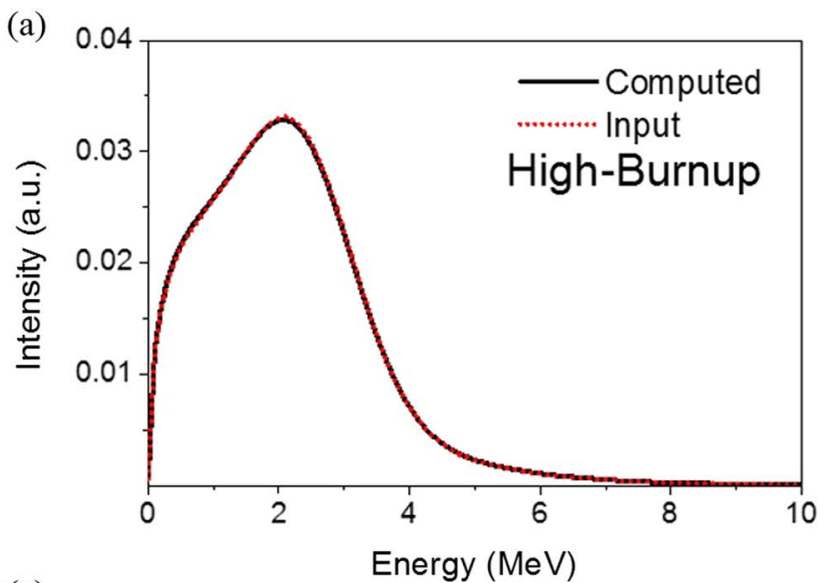

(c)

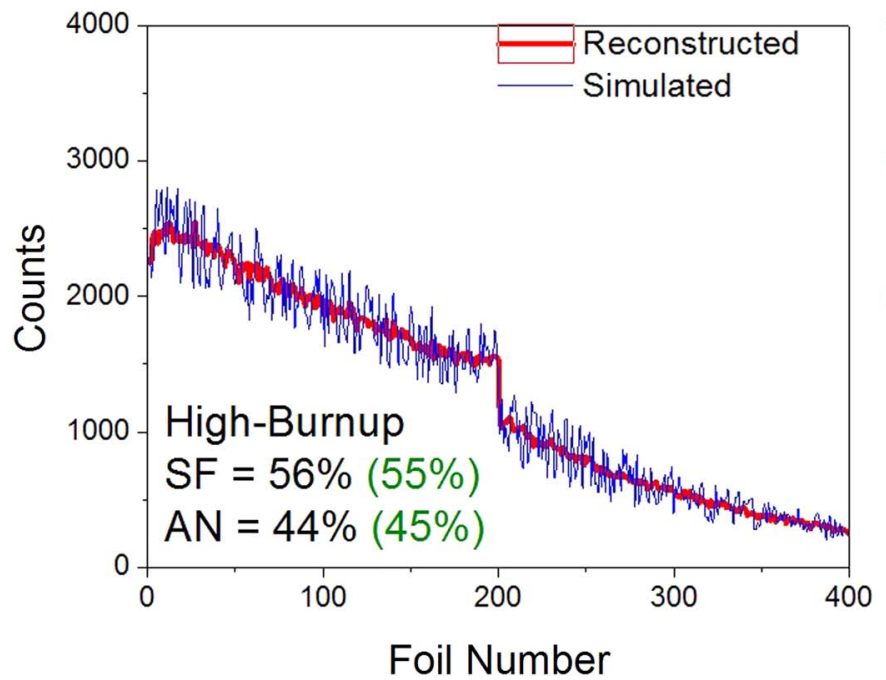

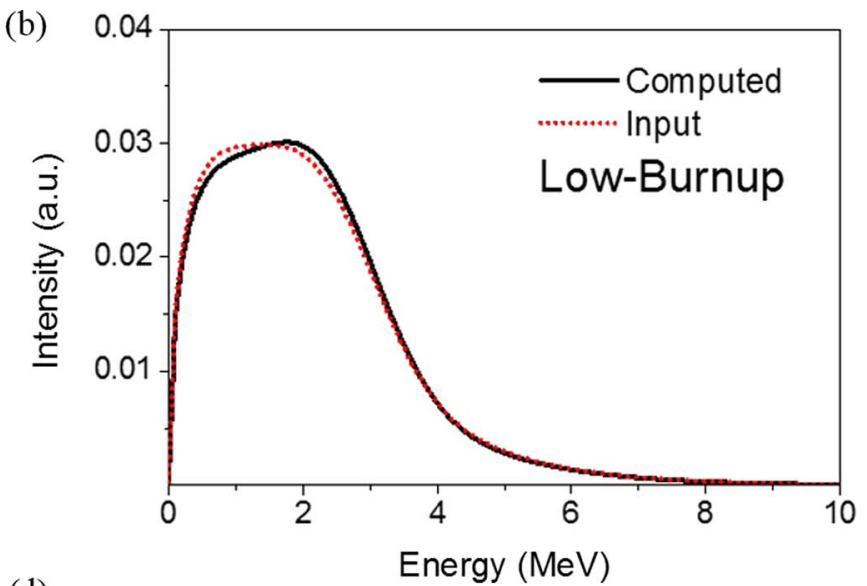

(d)

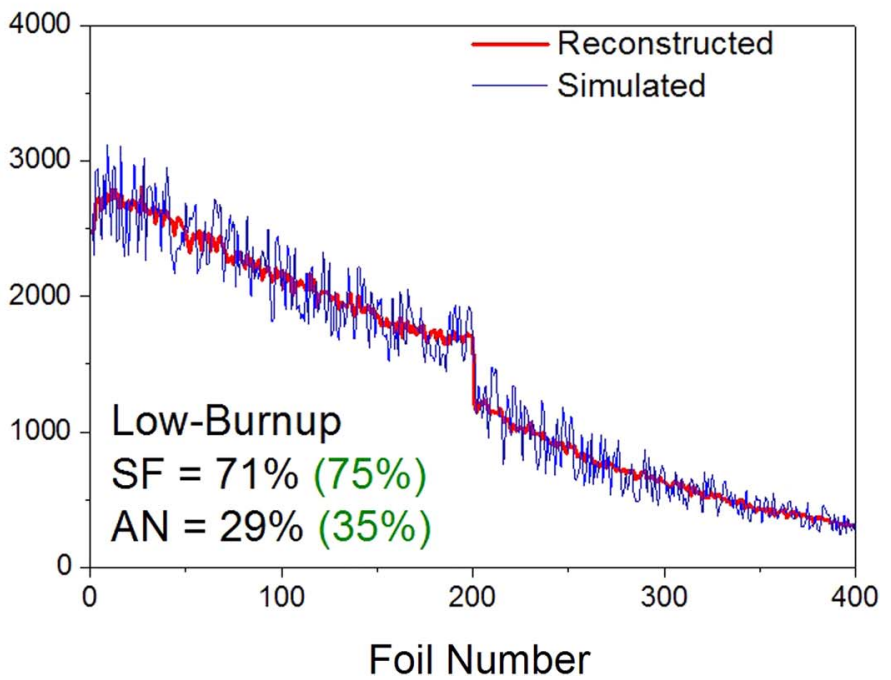

FIG. 8. Simulated detector response (blue graphs) from high-burnup and low-burnup MOX fuel spectra, and their reconstruction as linear combination of the $\mathrm{SF}$ and AN spectral components. The least-square fitted fractions of the two spectral contributions are compared (given in black in the lower left corner) to the input values (green).

energy-selective converter to distinguish the relative contributions of SF and AN emission in a composite spectrum.

Finally, Figure 8 shows examples of simulated detector response (blue graphs in Figures 8(c) and 8(d)) for typical high-burnup and low-burnup MOX fuel spectra, and the reconstruction of the total MOX spectra (MOX) as linear combination of the SF and AN spectral responses (black graphs in Figures 8(a) and 8(b))

$$
\mathrm{MOX}=\mathrm{a}^{*} \mathrm{SF}+\mathrm{b}^{*} \mathrm{AN},
$$

where $a$ and $b$ are the relative magnitude coefficient of the spectral components SF and AN, respectively.

The computation of the relative amplitude of the two spectral components was performed by the standard least square algorithm of Matlab. The uncertainty in the computed fraction of the two spectral components is of the order of a few percent, only considering counting statistics. Values of the spectral amplitude coefficients computed from analysis of simulation data are given in Figures 8(c) and 8(d) (in black) and are compared to input data (in green): for instance, the relative amplitude of the high-burnup fuel from the analysis of the simulated response functions was of the order of $56 \%$ compared to the $55 \%$ of the input value. Though the examples depicted in Figure 8 were processed with a relatively low counting statistics, the reconstructed fraction of SF and AN spectral contributions deviated from the input values by only a few percent.

\section{CONCLUSION AND DISCUSSION}

This work introduced a novel fast-neutron imaging detector concept, capable of energy-selective imaging or, in a more complex setup for neutron imaging spectroscopy using fewchannel unfolding techniques. The conceptual design of the detector is based on multiple energy-selective stacks of converter foils, aligned perpendicular to the direction of the impinging neutron beam, coupled to a position-sensitive charge readout (based on MWPC or a MPGD technology). Each stack consists of a multitude of converter foils, where each foil is a double layer made of: a polyethylene radiator part for neutron-to-proton conversion and a hydrogen-free coating for energy selective proton cut-off. The converter foils are separated by sub-millimeter gas gaps where neutron-induced recoil protons, escaping the foils, deposit energy in the form 
of ionization electrons. These charges are drifted, multiplied, and localized by the attached position-sensitive charge multiplier and readout system. Each stack has a different and well selected sensitivity to a specific fast-neutron energy range, according to the thickness of its hydrogenous radiator and its energy-selective coating. Recording the responses to a given neutron spectrum from different stacks allows the reconstruction of the impinging neutron fluxes by unfolding techniques. The two dimensional imaging capability of the detector allows for simultaneous measurement of the spectrum and its 1-dimensional spatial and spectral flux distribution.

The novel detector concept aims at two potential fields of application: one-dimensional energy-selective imaging and fast-neutron spectroscopy. The geometrical design of the converter should be tailored according the specific application; in this work, we discussed the design of two detector models derived from detailed Monte Carlo simulation, which incorporated the computation of accurate detector response functions.

Within the context of energy-selective imaging, the computer-simulation results show that the proposed concept provides a novel method of separating contributions of different energy ranges to the images. We have discussed a potential application in nuclear safeguarding with the aim of determining the plutonium content in MOX fuels. Other potential applications are detection and analysis of special nuclear materials for preventing smuggle of improvised nuclear weapons and disarmament verification transuranic waste characterization, material safeguarding, and facility decontamination and decommissioning. Energy-selective neutron imaging is also an important diagnostic instrument for monitoring the neutron emission rate and spatial distribution from plasma reactions, which provides information about the spatial distribution of the ion temperature and the degree to which reaction products have been incorporated in the plasma. ${ }^{24,25}$ The next phase of the project will focus on the development of a small imaging detector prototype comprised of few energyselective converter prototypes coupled to a $10 \times 10 \mathrm{~cm}^{2}$ position-sensitive charge readout. The latter will be based on THick Gaseous Electron Multiplier (THGEM). The operation of a multi-foils converter (no energy-selective coating) coupled to a THGEM based detector has been already tested and reported elsewhere. ${ }^{12}$ The main technological challenge is the production of a fine converter structure, comprising of robust sub-millimeter polyethylene foils coated with energyselective layer; presently various promising technologies are investigated, including 3-dimensional printing. A tests of a first converter prototype coupled with the THGEM-based detector prototype is underway, including a detailed study of the influence of material properties on the electron transfer efficiency in the small gas gaps between the converter foils.
${ }^{1}$ I. S. Anderson, R. McGreevy, and H. Z. Bilheux, Neutron Imaging and Applications: Neutron Application and Techniques (Springer Science + Business Media, LLC, 2009).

${ }^{2}$ I. Mor et al., "Fast-neutron imaging spectrometer based on liquid scintillator loaded capillaries," J. Instrum. 7, C04021 (2012).

${ }^{3}$ I. Imaida et al., "A new tracking satellite-borne solar neutron detector," Nucl. Instrum. Methods Phys. Res., Sect. A 421, 99-112 (1999).

${ }^{4}$ J. M. Ryan et al., "A scintillating plastic fiber tracking detector for neutron and proton imaging and spectroscopy," Nucl. Instrum. Methods Phys. Res., Sect. A 422, 49-53 (1999).

${ }^{5}$ E. Bogolubov, A. Koshelev, V. Mikerov, and A. Sviridov, "Feasibility of a large area detector for fast neutron imaging," J. Instrum. 7, C03034 (2012).

${ }^{6} \mathrm{~A}$. Breskin et al., "A novel liquid-xenon detector concept for combined fast-neutrons and gamma imaging and spectroscopy," J. Instrum. 7, C06008 (2012).

${ }^{7}$ J. R. D. Copley and T. J. Udovic, "Neutron time-of-flight spectroscopy," J. Res. Natl. Inst. Stand. Technol. 98, 71-87 (1993).

${ }^{8} \mathrm{I}$. Mor et al., "High spatial resolution fast-neutron imaging detectors for pulsed fast-neutron transmission spectroscopy," J. Instrum. 4, P05016 (2009).

${ }^{9}$ V. Dangendorf et al., "Detectors for energy-resolved fast neutron imaging," Nucl. Instrum. Methods Phys. Res., Sect. A 535, $93-97$ (2004).

${ }^{10} \mathrm{~F}$. Murtas et al., "Triple GEM gas detectors as real time fast neutron beam monitors for spallation neutron sources," J. Instrum. 7, P07021 (2012).

${ }^{11}$ M. Cortesi, R. Zboray, R. Adams, V. Dangendorf, and H.-M. Prasser, "Concept of a novel fast neutron imaging detector based on THGEM for fanbeam tomography applications," J. Instrum. 7, C02056 (2012).

${ }^{12} \mathrm{M}$. Cortesi et al., "Development of large-area THGEM detectors for investigation of thermal-hydraulic phenomena using neutron imaging," J. Instrum. 8, C10009 (2013).

${ }^{13}$ MCNPX Home Page, see https://mcnpx.lanl.gov.

${ }^{14} \mathrm{M}$. Titov, "Perspectives of micro-pattern gaseous detector technologies for future physics projects," in Proceedings of the CMS Workshop, Perspectives on Physics and on CMS at Very High Luminosity, May 2012, Alushta, Ukraine (Zarubin, Dubna, Ukraine, 2013).

${ }^{15}$ F. Sauli, "GEM: A new concept for electron amplification in gas detectors," Nucl. Instrum. Methods Phys. Res., Sect. A 386, 531-534 (1997).

${ }^{16}$ A. Breskin et al., "A concise review on THGEM detectors," Nucl. Instrum. Methods Phys. Res., Sect. A 598, 107-111 (2009).

${ }^{17}$ Y. Giomataris, P. Rebourgeard, J. P. Robert, and G. Charpak, "MICROMEGAS: A high-granularity position-sensitive gaseous detector for high particle-flux environments," Nucl. Instrum. Methods Phys. Res., Sect. A 376, 29-35 (1996).

${ }^{18}$ V. Fiorentini and G. Gulleri, "Theoretical evaluation of zirconia and hafnia as gate oxides for si microelectronics," Phys. Rev. Lett. 89, 266101 (2002).

${ }^{19} \mathrm{M}$. Cortesi et al., "First studies of electron transport along small gas gaps of novel foil radiation converters for fast-neutron detectors," J. Instrum. 8, P01016 (2013).

${ }^{20} \mathrm{M}$. Cortesi et al., "THGEM operation in $\mathrm{Ne}$ and Ne/CH4," J. Instrum. 4, P08001 (2009).

${ }^{21}$ D. L. Chichester et al., "Neutron emission characteristics of two mixedoxide fuels: Simulations and initial experiments," Technical Report, Idaho National Laboratory (INL), 2009.

${ }^{22}$ B. E. Watt, "Energy spectrum of neutrons from thermal fission of U235," Phys. Rev. 87, 1037-1041 (1952).

${ }^{23}$ T. Zak et al., "Neutron spectroscopy of plutonium oxide using matrix unfolding approach," Nucl. Instrum. Methods Phys. Res., Sect. A 622, 191195 (2010).

${ }^{24}$ W. Schneider et al., "Neutron diagnostics at the Wendelstein 7-X stellarator," J. Instrum. 7, C03025 (2012).

${ }^{25} \mathrm{R}$. Wolf et al., "From Wendelstein 7-X to a stellarator reactor," Plasma Fusion Res. 5, S1011 (2010). 